Oikos 119: 3-9, 2010

doi: $10.1111 / \mathrm{j} .1600-0706.2009 .17613 . x$,

(C) 2009 The Authors. Journal compilation (C) 2009 Oikos Subject Editor: Franz Weissing. Accepted 29 June 2009

\title{
The economics of evolution: Henry Ford and the Model T
}

\author{
Robert A. Laird and Thomas N. Sherratt \\ R. A. Laird, Dept of Biological Sciences, Univ. of Lethbridge, Lethbridge, Alberta, T1K 3M4, Canada. - T. N. Sherratt (sherratt@ \\ ccs.carleton.ca), Dept of Biology, Carleton Univ., Ottawa, Ontario, KIS 5B6, Canada.
}

\begin{abstract}
For the past 30 years evolutionary biologists have used a fictional tale about engineer and businessman Henry Ford to help illustrate the undesirability of over-design. Thus, on discovering that kingpins were rarely damaged in scrapped Model T automobiles, Henry Ford is alleged to have concluded that the kingpins were unnecessarily durable and asked that they be built to a cheaper specification. The general lesson that has been drawn from this tale is that natural selection will act to equalize the mortality risks accruing from damage to each part of the organism's body. Yet it is well known, at least as far as humans are concerned, that death is more likely to be attributed to a failure of some organs compared to others. To understand why this might be so, we use some graphical and mathematical models to show that even if all body organs were equally important to survivorship, then the optimal investment solution that maximizes wholeorganism longevity will typically not be the solution that equalizes the fail-times of the individual organs. As with natural organisms, the key to any optimal investment policy in multi-component systems is understanding what 'bang you can get for your buck'. Moreover, we use a specific model to show that, even following selection to ameliorate the effects of damage, those body parts that receive more damage are still more likely to be the ultimate cause of death - that is, there is 'under-compensation'. Therefore, the decision to make the kingpins more cheaply should not have been based only on the fact they rarely cause car failure compared to other car components. Such arguments wrongly assume that if one car part (or body part) is less durable than the others, then it will always be the reason for any future breakdown (or death).
\end{abstract}

On 27 September 1908, the first Model T Ford was wheeled off the production line in Detroit, Michigan. It was a car built and priced to be accessible to the masses, and "helped put America on wheels". Since this auspicious event, the Model $\mathrm{T}$ has attracted numerous myths and one-liners (Henry Ford: "Any customer can have a car painted any color that he wants so long as it is black", Ford and Crowther 1922). Indeed, for the past few decades evolutionary biologists have repeatedly invoked a story centering on the business acumen of Henry Ford to help explain why organisms should not be 'over-designed'. As far as we can tell Nicholas Humphrey (1983) made up the story, although a similar argument appeared in Maynard Smith's (1962) seminal review on the causes of senescence, so it may have an even longer folk history. Humphrey introduced the story as follows:

\footnotetext{
"Henry Ford, it is said, commissioned a survey of the car scrap-yards of America to find out if there were parts of the Model T Ford which never failed. His inspectors came back with reports of almost every kind of failure: axles, brakes, pistons - all were liable to go wrong. But they drew attention to one notable exception, the kingpins of the scrapped cars invariably had years of life left in them. With ruthless logic Ford concluded that the kingpins on the Model $T$ were too good for their job and ordered that in future they should be made to an inferior specification".
}

The story seems to make excellent economic sense. Why spend good money on something that will rarely if ever fail? Surely under these conditions the quality of the kingpins could be reduced, without drastically affecting the longevity of the car. Since its introduction by Humphrey, the story has been used by some of the best science writers in the world including Richard Dawkins (1995), John Barrow (1995), and Jared Diamond (1997) to argue that natural selection should, and does, act to ensure that organisms' bodies have no weak links. Dawkins uses the example of bones, suggesting that natural selection will act to "achieve the ideal of making every bone equally likely to break". However, he also noted an important caveat, namely, that some bones are more important to survival than others, implying that rather than equalizing breakage rates, selection will act to equalize the mortality risks accruing from damage to each part of the organism's skeleton.

Here we take the opportunity of the recent centenary of the Model $\mathrm{T}$ to raise even more fundamental doubts over the lessons that one can draw from the tale, and to offer a more complete solution based on the principles of "quantitative evolutionary design" (Diamond 2002). First, we show graphically that when resources can be diverted to different body parts with constant failure rates, then the allocation strategy that maximizes whole-organism longevity does not involve equalizing failure rates among components but rather the instantaneous rate of change 
of failure rates of components per unit resource - a "marginal value" theorem (Charnov 1976, Perrin 1992). The underlying reason for this is clear - if you can gain a greater increase in overall organism longevity (and thereby fitness, assuming unaltered fertility schedules) by allocating a given resource to component $\mathrm{A}$ compared to $\mathrm{B}$ then you should do so, even if component $\mathrm{A}$ is already less likely to fail. So, natural selection can readily (indeed will almost inevitably) generate organisms whose individual organs (or bones) differ in the probability that they ultimately cause death (also see Alexander 1997 for related arguments about 'safety factors').

Second, we provide some specific examples to build upon the above graphical arguments, using reliability theory from engineering (Barlow and Proschan 1965) and a pair of simple simulation models to guide us. Third, we present data which show that certain body parts - such as the heart and lungs - in real organisms are far more likely to be responsible for death than other body parts. Intriguingly, these are parts of the body that, despite natural selection, appear intrinsically more likely to get damaged than others, and we use our above model to help explain why 'undercompensation' might be particularly prevalent in these bodily components.

\section{Graphical arguments}

In this section, we explore the case of simplified organisms that are composed of two components ('Component 1' and 'Component 2') with instantaneous, age-independent, constant failure rates of $\mathrm{k}_{1}$ and $\mathrm{k}_{2}$. This is one possible manifestation of Alexander's (1997) concept of a 'mixed chain' system. If there are no other sources of mortality, then the whole-organism instantaneous mortality rate is simply $\mathrm{k}_{1}+\mathrm{k}_{2}$ (such that individuals die for one reason, or the other). Consider the situation in which $\mathrm{k}_{1}$ and $\mathrm{k}_{2}$ are decreasing functions of the amount of resources invested in them. If an organism has a fixed amount of resources that it can invest to its two components, it can allocate a certain percentage to Component 1 and the balance to Component 2. How should an organism allocate its resources?

All else being equal, natural selection should optimize the allocation between the two components by minimizing the overall mortality rate of $\mathrm{k}_{1}+\mathrm{k}_{2}$. Since mortality rates in this example are constant, then minimizing the overall mortality rate will maximize the organism's longevity. The optimal allocation strategy will depend critically on how $\mathrm{k}_{1}$ and $\mathrm{k}_{2}$ change as functions of the percentage of the available resources that each is allocated. In some scenarios the optimal allocation strategy will also be the one that equalizes the failure rates of the components; for example, if the failure rates of the components are identical functions of the level of investment in them (Fig. 1a). However, in many other situations, the optimal allocation strategy leaves one component weaker than the other (Fig. 1b-e). In this particular situation, the optimal allocation strategy is typically (linear relationships aside) the one that equalizes the marginal failure rates rather than the absolute failure rates (Fig. 1a, 1b, 1e) - in other words, organisms should invest resources where they have the most to gain.
We do not know the specific forms of the relationships between failure rates and investment in different body parts. However, due to the myriad differences between the different parts of the body, it is almost inevitable that the failure rates of these parts will not be the same function of the amount of resources invested in them. Therefore, the optimal allocation strategy will naturally result in certain body parts being more likely to break than others. The corollary is clear: body parts that rarely breakdown (or indeed never break down; e.g. Component 1 in Fig. 1c, 1d) are not necessarily overbuilt.

\section{Mathematical (reliability theory) arguments}

We now develop a specific illustration of the above arguments using reliability theory. Reliability theory "is a body of ideas, mathematical models, and methods directed toward the solution of problems in predicting, estimating, or optimizing the probability of survival, mean life, or, more generally, life distribution of components or systems" (Barlow and Proschan 1965). Recently, reliability theory has been brought to bear upon the problem of biological senescence, both in non-evolutionary (Gavrilov and Gavrilova 2001) and in evolutionary contexts (Laird and Sherratt 2009). In this section, we adopt the evolutionary reliabilitytheoretic approach to help demonstrate how natural selection will typically not result in bodily components with equal expected durabilities, again contrary to the Model T story.

In our example, we again assume for simplicity that organisms die due to the failure of one of just two components called Block 1 and Block 2 (Fig. 2). Each block represents a critical life-support system for the organism. For example, in the context of cancer biology, the blocks might represent the tumor-suppression systems associated with a different lethal form of cancer arising in two different organs. When either block ceases to function, the organism dies. Crucially, we assume that each of the blocks comprise multiple redundant elements. These elements could be considered genes, or the products of genes, but like all body parts, the individual elements are subject to damage. Just as home-owners may invest in back-up generators, redundancy is a common means to buffer natural systems from damage (Nowak et al. 1997, Conant and Wagner 2003) and analogous multi-stage checks and balances are involved in tumor suppression (Armitage and Doll 1954, Frank 2004a, 2004b, 2004c). Let the number of elements in Block 1 and 2 be $\mathrm{n}_{1}$ and $\mathrm{n}_{2}$, respectively. In each discrete time step, each element in Block 1 is damaged with probability $\mathrm{d}_{1}$ and each element in Block 2 is damaged with probability $\mathrm{d}_{2}$. A block functions so long as at least one of its elements remains undamaged. In addition to block failure, organisms can also die for 'extrinsic' reasons, such as accidents, predation, and communicable diseases. Extrinsic mortality occurs with a probability $\mathrm{q}_{\mathrm{ext}}$ per time step.

Under these conditions, and assuming that the two intrinsic and one extrinsic mortality sources act independently, the expected probability of survivorship to time $t$ is given by: 


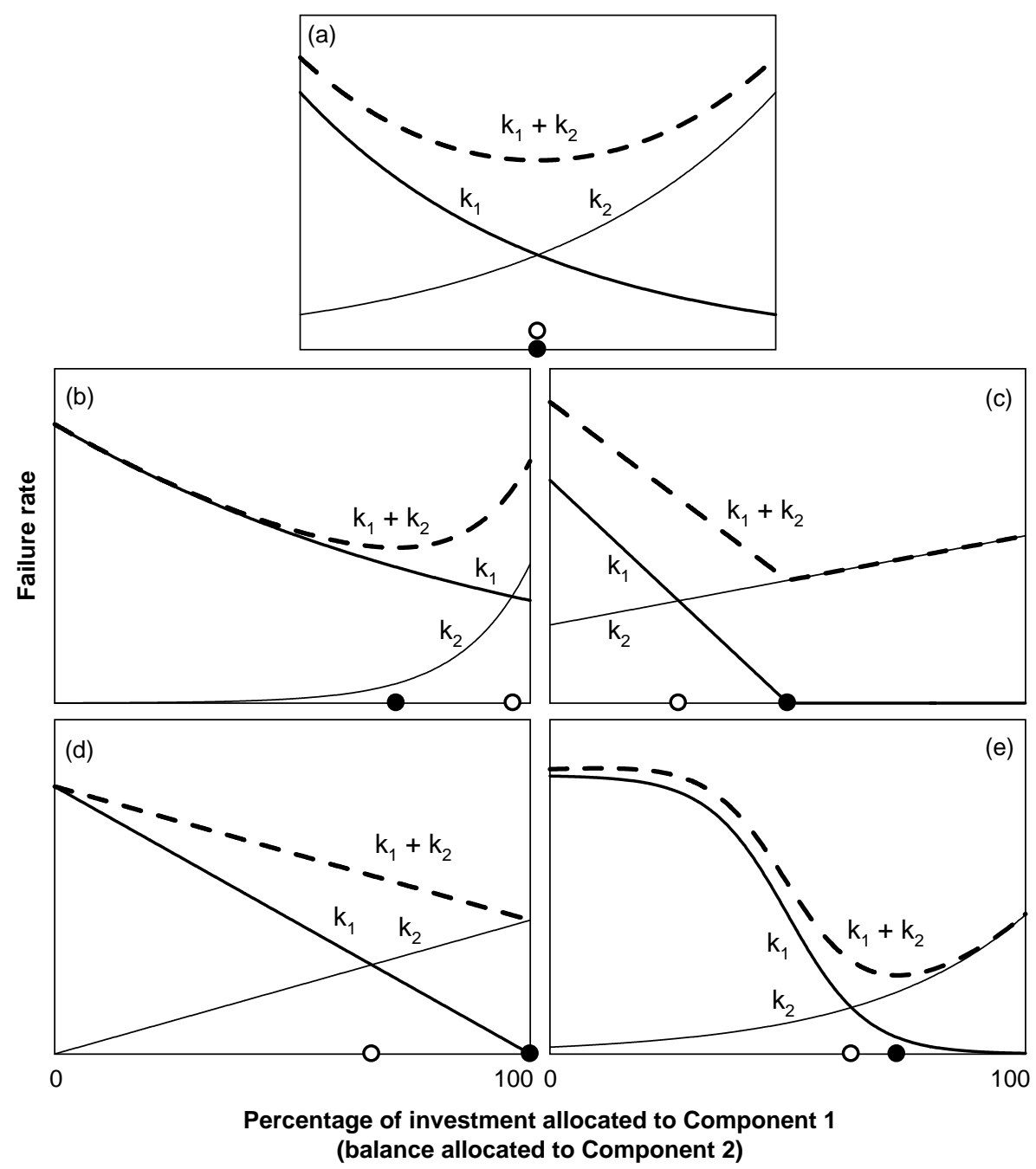

Figure 1. Allocation strategies in a two-component system in which both components have constant instantaneous failure rates, and there are no other sources of mortality. Panels show possible examples of the failure rates of Component 1 ( $\mathrm{k}_{1}$; thick solid lines), Component 2 ( $\mathrm{k}_{2}$; thin solid lines), and the overall mortality rate of the whole organism $\left(\mathrm{k}_{1}+\mathrm{k}_{2}\right.$; dashed lines), as functions of the percentage of available resources allocated to Component 1 (and assuming that the balance is allocated to Component 2). The failure rates are assumed to be constant through time. The open circles on the $\mathrm{x}$-axis represent the allocation strategy that equalizes $\mathrm{k}_{1}$ and $\mathrm{k}_{2}$; the closed circles represent the optimal allocation strategy that minimizes $\mathrm{k}_{1}+\mathrm{k}_{2}$. (a) the optimal allocation that minimizes $\mathrm{k}_{1}+\mathrm{k}_{2}$ also equalizes $\mathrm{k}_{1}$ and $\mathrm{k}_{2}$ (b-e) the optimal allocation that minimizes $\mathrm{k}_{1}+\mathrm{k}_{2}$ does not equalize $\mathrm{k}_{1}$ and $\mathrm{k}_{2}$; rather organisms adopting the optimal strategy are more likely to die from the failure of one component over the other.

$$
\begin{aligned}
\mathrm{l}_{\mathrm{t}}= & {\left[1-\left(1-\left(1-\mathrm{d}_{1}\right)^{\mathrm{t}}\right)^{\mathrm{n}_{1}}\right]\left[1-\left(1-\left(1-\mathrm{d}_{2}\right)^{\mathrm{t}}\right)^{\mathrm{n}_{2}}\right] } \\
& \times\left[\left(1-\mathrm{q}_{\mathrm{ext}}\right)^{\mathrm{t}}\right]
\end{aligned}
$$

where the first term in square brackets is the survivorship of Block 1, the second term in square brackets is the survivorship of Block 2, and the third term in square brackets is the survivorship associated with avoiding extrinsic mortality. Here the expected time to failure of Block 1 (assuming that death does not come first for any other reason) is $\sum_{\mathrm{t}=1}^{\infty}\left[1-\left(1-\left(1-\mathrm{d}_{1}\right)^{\mathrm{t}}\right)^{\mathrm{n}_{1}}\right]$, the expected time to failure of Block 2 (again under the condition that there is no other mortality source) is $\sum_{\mathrm{t}=1}^{\infty}[1-(1-(1-$ $\left.\left.\mathrm{d}_{2}\right)^{\mathrm{t}}\right)^{\mathrm{n}_{2}}$ ], where the expected longevity of whole organisms is $\sum_{\mathrm{t}=1}^{\infty} 1_{\mathrm{t}}$.

Consider the situation in which organisms must allocate limited resources to the redundancy of their two blocks. Specifically, let $\mathrm{N}=\mathrm{n}_{1}+\mathrm{n}_{2}$ be the total number of elements that an organism has at its disposal. In this case, the optimal allocation of elements that maximizes whole-organism longevity will typically not be the same as the allocation that equalizes the fail-times of Blocks 1 and 2. Indeed, in this reliability example, one must assume $d_{1}=d_{2}$ in order for the optimal allocation to result in the equalization of the expected longevities of the blocks. Considering that this assumption will frequently be violated (since some parts of the body are intrinsically more likely than others to experience damage), it is likely that natural selection will often result in body parts with different fail-times, rather than equalized fail-times - at least insofar as longer-lived individuals have greater fitness than shorter-lived individuals.

For example, in Fig. $3\left(\mathrm{~N}=20, \mathrm{~d}_{1}=0.2, \mathrm{~d}_{2}=0.1\right.$, $\left.\mathrm{q}_{\mathrm{ext}}=0.1\right)$, the allocation of elements that maximizes whole-organism longevity (dotted black line) differs from 


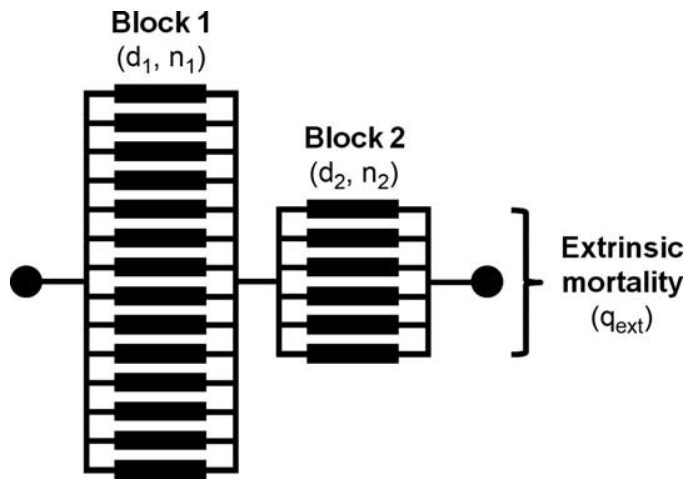

Figure 2. Schematic representation of an individual with a twoblock life-support system, within the framework of reliability theory. For example, in the context of cancer biology, the blocks could represent the suppression system for a specific type of tumor and the elements could represent redundant tumor-suppression loci. Block 1 has $\mathrm{n}_{1}$ elements and Block 2 has $\mathrm{n}_{2}$ elements (in this example, $\mathrm{n}_{1}=14, \mathrm{n}_{2}=6$ ). In each time step, every element in Block 1 has a probability $\mathrm{d}_{1}$ of being damaged and every element in Block 2 has a probability $\mathrm{d}_{2}$ of being damaged. When an element is randomly damaged, the path on which that element is situated is broken. As long as there is at least one continuous path between the circles, the organism survives (e.g. it does not develop a tumor and die of cancer); unless it dies due to 'extrinsic' causes with probability $\mathrm{q}_{\mathrm{ext}}$ (encompassing predation, communicable disease, natural disasters, etc.). However, once all the elements of a given block are broken, the organism dies due to bodily failure (e.g. it develops the tumor type represented by the failed block, and dies of cancer).

the allocation that equalizes block fail-time (short-dashed black line). Specifically, although the optimal allocation of elements favours the more damage-prone block (in this case, Block 1; compare long-dashed and dotted black lines), it does not do so to a degree that equalizes block fail-times (compare dotted and short-dashed black lines). Rather, optimally allocating organisms still have Block $1 \mathrm{~s}$ with much shorter expected fail-times than Block 2 s, and are still much more likely to die due to the failure of the more damage-prone block. Yet, Block 2 is not 'overbuilt' in any meaningful sense; it simply receives damage at a lower rate. Note also that unlike the example of the graphical arguments above, where the mortality rates were constant through time (Fig. 1), in this slightly more complex scenario the rates of change of the fail-times are not equalized either (Fig. 3).

We further investigated the allocation problem using a simple discrete-time simulation. The simulation started with a population of 1000 individuals, each with a random allocation of $\mathrm{N}=20$ elements to two blocks. Each generation was composed of multiple time steps. As above, in each time step, each element in each individual's Block 1 was damaged with a probability of $d_{1}$, each element in each individual's Block 2 was damaged with a probability of $\mathrm{d}_{2}$; also, in each time step, each individual died due to extrinsic mortality with a probability of $\mathrm{q}_{\mathrm{ext}}$. Every time an individual survived a time step, it reproduced (asexually), adding a single prospective offspring to the next generation. Thus, lifetime reproductive output (hence fitness in this discrete-time simulation) was set to exactly equal longevity. The offspring were identical to their parents, except that

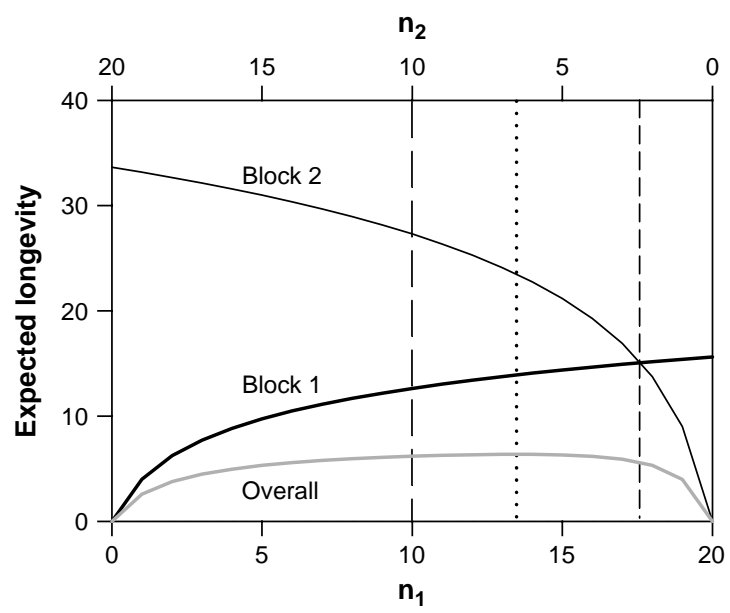

Figure 3. The expected fail-time (i.e. block 'durability', provided the organism has not died due to another cause) of Block 1 (thick solid black line) and Block 2 (thin solid black line) and wholeorganism ('overall') longevity (thick grey solid line) as a function of $\mathrm{n}_{1}$ (increases left-to-right) and $\mathrm{n}_{2}$ (increases right-to-left) for the two-block reliability theory example. The damage probabilities per time step of the elements in Block 1 and Block 2 were set at $d_{1}=$ 0.2 and $\mathrm{d}_{2}=0.1$ respectively. The total number of elements available was set at $\mathrm{N}=\mathrm{n}_{1}+\mathrm{n}_{2}=20$ and the extrinsic mortality was $\mathrm{q}_{\mathrm{ext}}=0.1$. The vertical long-dashed black line represents equal allocation to Blocks 1 and 2. The vertical short-dashed black line represents the allocation that equalizes the expected fail-times of Blocks 1 and 2. The vertical dotted black line represents the optimal allocation that maximizes whole-organism longevity.

there was a mutation probability of 0.0001 of reallocating an element between Block 1 and Block 2 (the direction of this shift was determined randomly, with the restriction that $0<\mathrm{n}_{1}<\mathrm{N}$ and $\left.0<\mathrm{n}_{2}<\mathrm{N}\right)$. At the end of a generation (i.e. when all the individuals had died due to block failure or extrinsic causes), 1000 of the offspring were randomly chosen to make up the next generation. Ten replicates of the simulation were run, each for 10000 discrete generations.

The results of this simulation are shown in Fig. 4a and $4 \mathrm{~b}$ (for the same parameter values as Fig. 3). The relative contribution of extrinsic mortality to total mortality at first rises as organisms evolve ways to reduce their intrinsic mortality by better balancing their allocations $n_{1}$ and $n_{2}$ to each block (Fig. 4b). As expected from Fig. 3 however, natural selection ended up under-compensating for Block 1 's greater damage rate, producing an allocation of redundant elements between Blocks 1 and 2 close to the optimal split given in Fig. 3. Indeed, even after 10000 generations, although there were, on average, more elements allocated to Block 1 (Fig. 4a), the probability of dying due to the failure of Block 1 still greatly exceeded the probability of dying due to the failure of Block 2 (Fig. 4b).

One might argue that these results were due to the implicit cost of redundancy, and that if elements were costfree and not subject to the tradeoff embodied by the equation $\mathrm{N}=\mathrm{n}_{1}+\mathrm{n}_{2}$, then natural selection would be free to equalize block fail-time. To test whether blocks would evolve equalized longevities when their elements were costfree, we re-ran the above simulation with some minor changes. First, individuals all started with one element in 
Fixed N

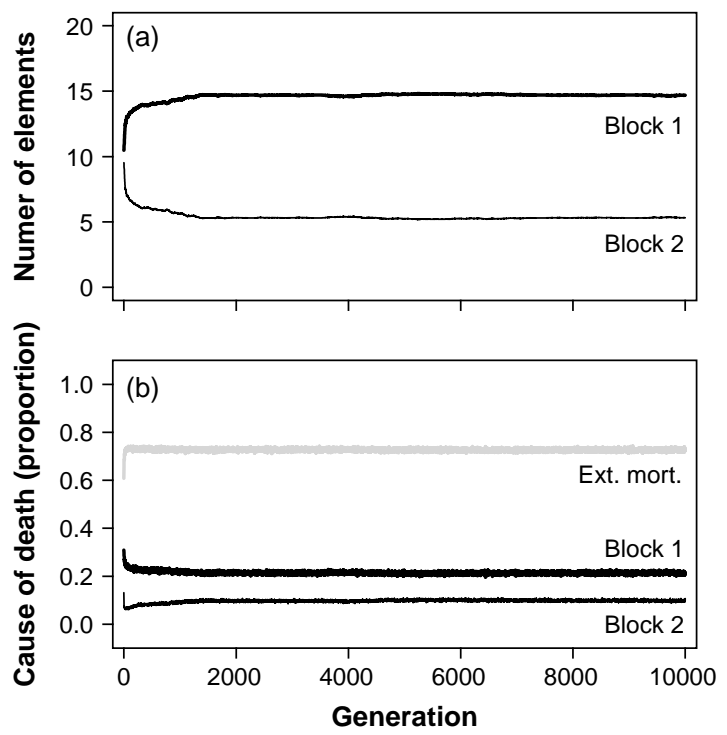

Non-fixed N
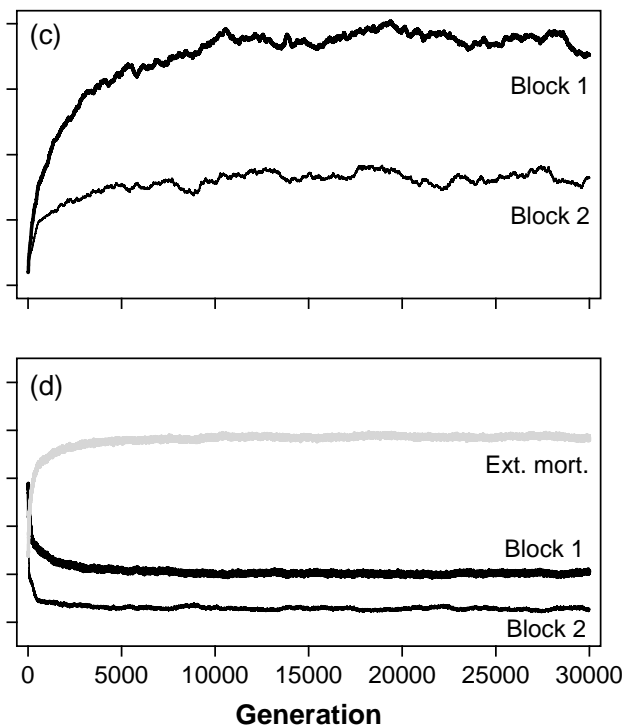

Figure 4. (a, b) simulation results when $\mathrm{N}$ is fixed. The results show the average of 10 replicates of 1000-individual populations. Parameter values: $\mathrm{N}=20, \mathrm{~d}_{1}=0.2, \mathrm{~d}_{2}=0.1, \mathrm{q}_{\mathrm{ext}}=0.1$, probability of reallocation of elements between blocks of 0.0001 per offspring. (a) the number of elements of Block 1 (thick black line) and Block 2 (thin black line) that evolve over 10000 simulated generations. (b) the proportion of individuals that die due to the failure of Block 1 (thick black line), the failure of Block 2 (thin black line), and extrinsic mortality (grey line). (c, d) simulation results when $\mathrm{N}$ is not fixed. The results show the average of 10 replicates of 1000 -individual populations. Parameter values: $\mathrm{d}_{1}=0.2, \mathrm{~d}_{2}=0.1, \mathrm{q}_{\mathrm{ext}}=0.1$, probability of increasing $\mathrm{n}_{1}$ and $\mathrm{n}_{2}=0.001$ per offspring, probability of decreasing $\mathrm{n}_{1}$ and $\mathrm{n}_{2}=0.01$ per offspring. (c) the number of elements of Block 1 (thick black line) and Block 2 (thin black line) that evolve over 30000 simulated generations. (d) the proportion of individuals that die due to the failure of Block 1 (thick black line), the failure of Block 2 (thin black line), and extrinsic mortality (grey line).

each of their two blocks. Second, there was no constraint on the total number of elements allowed; $\mathrm{N}$ was not fixed (and, therefore, elements were cost-free). Third, mutations did not result in a reallocation of elements between Blocks 1 and 2. Rather, mutations occurred independently between the blocks, with a probability of increasing the number of elements in a block of 0.001 and a probability of decreasing the number of elements in a block of 0.01 (the biased mutation rates account for the fact that mutations are more likely to be deleterious than beneficial, Eyre-Walker and Keightley 2007). Fourth, we ran the 10 replicates for 30000 generations instead of 10000 , because it took longer for the number of elements in the blocks to level-off. Other than these alterations, we used the same parameter values as in the first simulation.

While longevity increases with both $\mathrm{n}_{1}$ and $\mathrm{n}_{2}$, it does so in a decelerating fashion (such that increasing $\mathrm{n}_{1}$ and $\mathrm{n}_{2}$ provides an increasingly smaller survival advantage if the organism is likely die from predation before the benefits of this redundancy can be realized). Therefore, as expected, both Block 1 and Block 2 evolved multiple elements, but eventually the number of elements leveled off (Fig. 4c) as a form of mutation-selection balance (Medawar 1952) was reached. On average, Block 1 evolved more elements than Block 2, in order to compensate for Block 1's greater damage rate (Fig. 4c). However, even with cost-free elements, this compensation was incomplete. As with the first simulation, even after 30000 generations, individuals were still much more likely to die due to the failure of Block 1 than Block 2 (Fig. 4d).
These more explicit mechanistic models show that in systems whose components' reliabilities are governed by multiple, redundant parts, natural selection will generally not equalize the durability of those components.

\section{Observational data}

Despite occasional assertions to the contrary, evolution has not shaped different components of human bodies to 'fall apart' at the same time. We can see this most directly by noting that, for example, in humans heart failure is far more likely to be the cause of death than liver failure (Fig. 5). Likewise, Currey (1984) analysed records of accidents in horse racing and found that horses' distal leg bones were much more likely to suffer fatigue fractures than proximal ones. Why should this be so? We suggest that the very functions different body parts perform render some parts more likely to experience damage than others. Similarly, some body parts may be more expensive to maintain than others. Natural selection will act to ameliorate the effects of damage, but the differing returns on investment means that organs are not likely to be rendered equally likely to cause death. Put another way, why invest resources to improve the durability of the heart when one can get more return from investing in the liver? Indeed, Currey (1984) suggested that the difference in the likelihood of distal and proximal leg bones of horses suffering fractures could be explained by the higher energetic running demands imposed by any increase in the mass of the distal part of the leg; alternatively, distal 


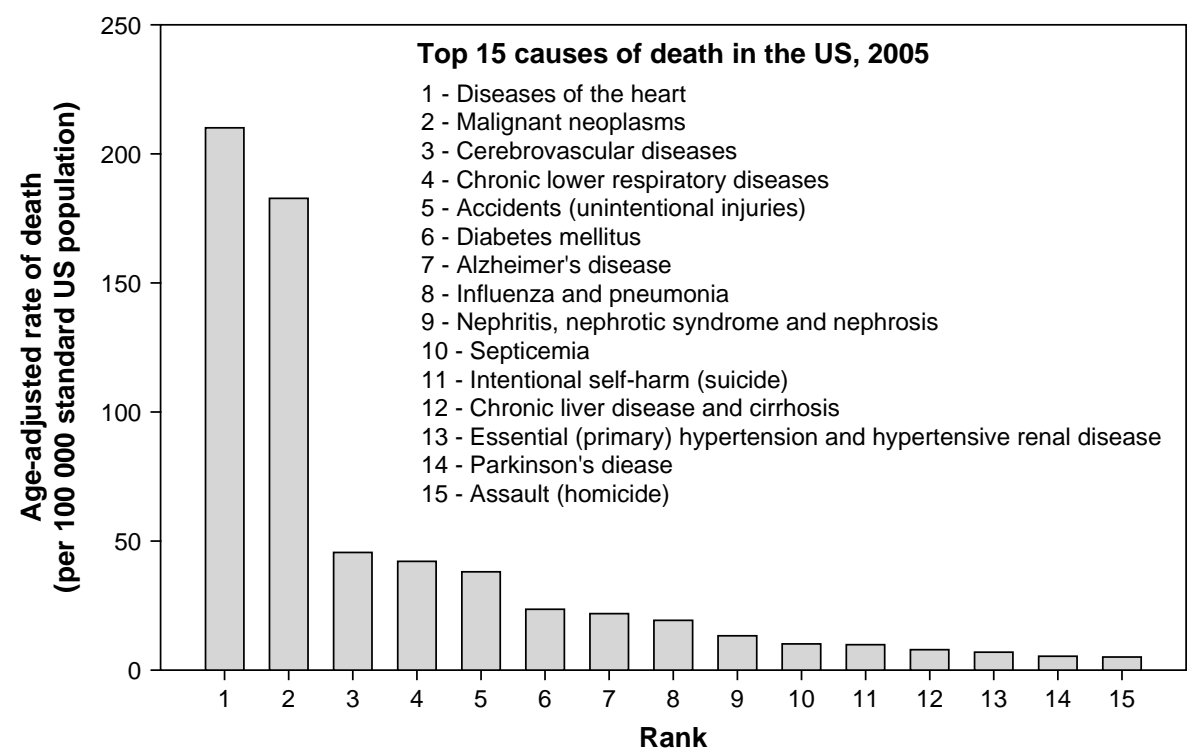

Figure 5. The top 15 causes of death in humans in the USA in 2005 (data from Kung et al. 2008).

leg bones may be more stress-prone, yet evolution has undercompensated for this, similar to our simulation examples.

\section{Problem, what problem?}

One might wonder why it is so easy to fall into the Model T Ford fallacy, in spite of (1) the history of 'marginal value' arguments in ecology (Charnov 1976, Perrin 1992), (2) detailed discussions of the closely related question of why different body parts have greater capacity-to-load ratios ('safety factors') than others (Alexander 1981, 1997, Diamond 2002), and (3) the exposure of evolutionary biologists to other economic fallacies such as the 'Concorde fallacy', (Dawkins and Brockmann 1980). Viewing an automobile or an organism as a linked chain of parts immediately leads to the conclusion that there should be no weakest links. As Diamond (2002) noted, "A simple argument in favour of equal capacities is that such a design would avoid wasting energy on high-capacity components whose high capacity could never be utilized because lowercapacity components in series would always limit the performance of the whole system." However, such a perspective implicitly assumes that the individual links in the chain are equal both in terms of the damage they receive, and in the costs of strengthening them. Moreover, it also implicitly assumes that if one link in the chain were weaker than the others, than this weak link, as opposed to its stronger brethren, would always be the source of chain failure. If any of these assumptions are violated, then the optimal solution is unlikely to involve equalizing the underlying rates of deterioration of separate links (Alexander 1997, Diamond 2002). Would Henry Ford have been better off leaving the brass kingpins alone? Maybe, but maybe not. After all, it is still possible to overbuild components (e.g. any sub-optimal allocation to $\mathrm{n}_{1}$ and $\mathrm{n}_{2}$ in Fig. 3). However, the decision to make the kingpins more cheaply should not be based simply on the fact they rarely cause car failure compared to other car components. As with natural systems, the key to an optimal investment policy is understanding what 'bang you can get for your buck'.

Acknowledgements - We thank the handling editor for his comments. Funding for this research was provided by the Natural Sciences and Engineering Research Council (Canada) and the Canada Foundation for Innovation.

\section{References}

Alexander, R. M. 1981. Factors of safety in the structure of animals. - Sci. Progr. 67: 109-130.

Alexander, R. M. 1997. A theory of mixed chains applied to safety factors in biological systems. - J. Theor. Biol. 184: 247-252.

Armitage, P. and Doll, R. 1954. The age distribution of cancer and a multi-stage theory of carcinogenesis. - Brit. J. Cancer 8: 1-12. (reprinted in Int. J. Epidemiol. 33: 1174-1179.)

Barlow, R. E. and Proschan, F. 1965. Mathematical theory of reliability. - Wiley.

Barrow, J. D. 1995. The artful universe: the cosmic source of human creativity. - Clarendon Press.

Charnov, E. L. 1976. Optimal foraging: the marginal value theorem. - Theor. Popul. Biol. 9: 129-136.

Conant, G. C. and Wagner, A. 2003. Duplicate genes and robustness to transient gene knock-downs in Caenorhabditis elegans. - Proc. R. Soc. Lond. B 271: 89-96.

Currey, J. D. 1984. The mechanical adaptations of bones. - Princeton Univ. Press.

Dawkins, R. 1995. River out of Eden. - Weidenfeld and Nicholson.

Dawkins, R. and Brockmann, H. J. 1980. Do digger wasps commit the Concorde fallacy? - Anim. Behav. 28: 892-896.

Diamond, J. 1997. Why is sex fun? The evolution of human sexuality. - Basic Books, New York.

Diamond, J. 2002. Quantitative evolutionary design. - J. Phys. 542: $337-345$. 
Eyre-Walker, A. and Keightley, P. D. 2007. The distribution of fitness effects of new mutations. - Nat. Rev. Genet. 8: 610-618.

Ford, H. and Crowther, S. 1922. My life and work (10th ed.). - Project Guttenburg E-Book.

Frank, S. A. 2004a. A multistage theory of age-specific acceleration in human mortality. - BMC Biol. 2: 16.

Frank, S. A. 2004b. Genetic variation in cancer predisposition: mutational decay of a robust genetic control. - Proc. Natl Acad. Sci. USA 101: 8061-8065.

Frank, S. A. 2004c. Age-specific acceleration of cancer. - Curr. Biol. 14: 242-246.

Gavrilov, L. A. and Gavrilova, N. S. 2001. The reliability theory of aging and longevity. - J. Theor. Biol. 213: 527-545.

Humphrey, N. 1983. Consciousness regained: chapters in the development of mind. - Oxford Univ. Press.
Kung, H. C. et al. 2008. Deaths: final data for 2005. National vital statistics reports; vol. 56 no. 10. - Natl Center Health Stat., Hyattsville, MD.

Laird, R. A. and Sherratt, T. N. 2009. The evolution of senescence through decelerating selection for system reliability. - J. Evol. Biol. 22: 974-982.

Maynard Smith, J. 1962. Review lectures on senescence. I. The causes of ageing. - Proc. R. Soc. Lond. B 157: 115-127.

Medawar, P. B. 1952. An unsolved problem of biology. - Western Printing Systems, Bristol.

Nowak, M. A. et al. 1997. Evolution of genetic redundancy. - Nature 388: 167-171.

Perrin, N. 1992. Optimal resource allocation and the marginal value of organs. - Am. Nat. 139: 1344-1369. 